\title{
Application of principal component analysis (PCA) and improved joint probability distributions to the inverse first-order reliability method (I-FORM) for predicting extreme sea states
}

\author{
Aubrey Eckert-Gallup ${ }^{1}$, Cédric Sallaberry, Ann Dallman, Vincent Neary \\ Sandia National Laboratories, Albuquerque, NM 87185, USA
}

\begin{abstract}
Environmental contours describing extreme sea states are generated as the input for numerical or physical model simulations as a part of the standard current practice for designing marine structures to survive extreme sea states. Such environmental contours are characterized by combinations of significant wave height $\left(H_{s}\right)$ and energy period $\left(T_{e}\right)$ or peak period $\left(T_{p}\right)$ values calculated for a given recurrence interval using a set of data based on hindcast simulations or buoy observations over a sufficient period of record. The use of the inverse first-order reliability method (I-FORM) is standard design practice for generating environmental contours. This paper develops enhanced methodologies for data analysis prior to the application of the I-FORM, including the use of principal component analysis (PCA) to create an uncorrelated representation of the variables under consideration as well as new distribution and parameter fitting techniques. These modifications better represent the measured data and, therefore, should contribute to the development of more realistic representations of environmental contours of extreme sea states for use in survivability analysis for marine structures.
\end{abstract}

\footnotetext{
${ }^{1}$ Corresponding author. Tel.:505-844-4508; Email: acecker@sandia.gov
} 
Keywords: Inverse FORM, Principal Component Analysis, Environmental Contours, Extreme Sea State Characterization.

\section{Introduction}

The current practice for designing marine structures to survive extreme sea states is to apply nonlinear time domain numerical simulations to predict structural response to a short-term extreme wave or wave group. Extreme wave design generally includes the following steps as outlined in Coe et al. (2014): (1) consideration of hindcast simulations or buoy observations of sufficient duration and appropriate location; (2) application of extreme value theory and models used for extrapolation to events more extreme than those observed in a shorter period of record; (3) generation of environmental contours consisting of pairs of significant wave height $\left(H_{s}\right)$ and either peak period $\left(T_{p}\right)$ or energy period $\left(T_{e}\right)$ that elicit extreme structural responses for a given return period; (4) identification of one or more extreme sea states, which can be used with a wave spectrum (often Pierson-Moscowitz or JONSWAP) to reconstruct time series data generated with a random-phase/amplitude model as input for numerical or physical model simulation (Berg, 2011). The energy period is widely used in wave energy applications (Lenee-Bluhm et al., 2011) and has been found to be more robust than the peak period due to a high sensitivity to spectral peak.

Vanem and Bitner-Gregersen (2014) summarize methods for generating environmental contours. These methods include the traditional inverse first-order reliability method (I-FORM) utilizing the Rosenblatt transformation (Rosenblatt, 1952), described in detail by Winterstein et al. (1993), and newer methods that avoid the Rosenblatt transformation by employing Monte Carlo simulations of a joint probability model (Huseby et al., 2013). Silva-González et al. (2013) use the Nataf transformation to capture the correlation between inputs and create environmental contours using the marginal distributions 
and correlation coefficients of the environmental variables in a particular problem of interest. The I-FORM continues to be standard design practice for generating environmental contours used for estimating extreme sea states of a given recurrence interval or return period (DNV, 2014). Environmental loads associated with these extreme sea states are used to design various marine structures, including ships (DNV, 2002), dynamic risers (DNV, 2001), position moorings (DNV, 2010a), offshore floating platforms (DNV, 2010b), and wave energy converters (WEC) (DNV, 2008).

The use of the traditional I-FORM is clearly developed in Haver (2009) and has been applied by Berg (2011) in accordance with the recommendations in the DNV standard on position mooring (DNV, 2010a). The first step of this basic application of the I-FORM described in these works includes fitting distributions and parameter models to the significant wave height $\left(H_{s}\right)$ and energy period $\left(T_{e}\right)$ observations such that their interdependency is captured. The treatment of this interdependency in Haver (2009) includes a joint probability distribution using parameters developed by Nygaard and Johannessen (2000). The second step of the traditional I-FORM application uses these fitted distributions to estimate extreme sea states by expressing the probability level for the return period of interest (e.g., 100 years) as the radius of a circular isoline in the standard normal space. This circle is defined by equiprobable points at discrete angular intervals determined by the combination of two variables with individually varying probabilities. The probabilities of each of these variables are used to evaluate the inverse of each of the previously defined probability distributions for the variables of the original input space in order to transform each point into the original space of interest, creating a final environmental contour.

Examples of this application can be found in Vanem and Bitner-Gregersen (2014). In some cases, environmental contours for a 100-year return period calculated using this traditional application fail to cover the observations taken over a relatively short (8 to 10year) period of record. This problem was addressed by Berg (2011) by inflating the contour 
resulting from the I-FORM using an arbitrary percentage value. The shortcomings of the environmental contours generated using the traditional methodology along with attempts to correct these problems as a post-processing step clearly show that this methodology requires further exploration and modification in order to generate more realistic contours.

Others have addressed the need to modify the treatment of input variables prior to the use of the I-FORM approach. For example, Forristall and Cooper (1997) use empirical orthogonal functions (EOFs) in order to create more appropriate design water current profiles using the I-FORM. In their paper, EOFs are used to simplify the time series data for ocean currents into a series of simpler modes, reducing the original vertical profile. These simplified input variables are then evaluated using the I-FORM to generate design current profiles. The use of EOFs by Forristall and Cooper (1997) shows that it is important to enhance methods for addressing the complexity of input data before applying the probability distribution fittings required by the I-FORM. The importance of capturing the correlations between input variables is also addressed by Silva-González et al. (2013). In the current work, input data complexity will be reduced using principal component analysis (PCA).

This paper describes techniques for improving the traditional I-FORM through principal component analysis and enhanced extreme value models for the subsequent uncorrelated random variables. Following the use of PCA to reduce the correlation between the input variables, the I-FORM is applied with modifications to the traditional distribution and parameter fitting models for the subsequent uncorrelated variables. These modifications are shown to contribute to the development of more accurate and reasonable representations of environmental contours for extreme sea states. Signficant wave height and energy period data collected at four buoys is used in this analysis: National Data Buoy Center (NDBC) 46212 offshore of Northern California in $40 \mathrm{~m}$ depth with 76608 hourly observations taken from January 22, 2004 to December 31, 2012, NDBC 46022, also offshore of Northern California in $675 \mathrm{~m}$ depth with 122467 hourly observations taken from 
January 1, 1996 to December 31, 2012, NDBC 51202 offshore of Oahu in $82 \mathrm{~m}$ depth with 105192 hourly observations taken from January 1, 2001 to December 31, 2012, and NDBC 46050 offshore of Oregon in $128 \mathrm{~m}$ depth with 126614 hourly observations taken from January 1, 1996 to December 31, 2012. The measured spectral data from these buoys was used to calculate significant wave height and energy period at each site.

This paper is organized as follows. Section 2 describes the use of PCA to create a set of uncorrelated variables that will be used to generate better-fitting environmental contours. Section 3 discusses an improved methodology for creating distribution and parameter models for the variables of interest so that the I-FORM can be applied. Section 4 details the application of the I-FORM to the problem under consideration and presents the results of the implementation of this methodology. Section 5 provides concluding remarks and describes recommendations for future work.

\section{Principal component analysis}

The concept of principal components was initially introduced by Pearson (1901) and more formally developed by Hotelling (1933). PCA provides a powerful transformation that works to reduce the dimensionality of a problem by considering that higher order components have a low impact on the variance of the data. The underlying goal of PCA is to develop a new orthogonal basis in which the variables will be (1) uncorrelated and (2) sorted such that the first variable represents the direction in which the data has the largest variance and each subsequent variable leads to the next largest variance. Traditionally, the variables in the new basis are called principal components while the values associated to each variable in this new basis for each point are called z-scores. The mathematical tools used to generate this new basis are based on classical linear (matrix) algebra applied to the covariance matrix, taking advantage of its structure as a square, symmetrical, and non-singular matrix (Jackson, 1991). The application of PCA to the current problem is 
mathematically analogous to the use of the empirical orthogonal functions utilized in Forristall and Cooper (1997) for the purpose of generating water current profiles.

\subsection{Motivation}

The wave data across four sites of interest was studied and compared as a first step in the process of improving upon the traditional application of the I-FORM. This study included the creation of a representation of data density in order to understand the underlying patterns and trends masked by a traditional scatterplot representation of the data. The density around each point was calculated based on the number of observations included within a fixed circular neighborhood with a radius $\epsilon$ defined by $\epsilon=2000 / N$ where $N$ is the number of points in each dataset. Examples of the data density calculated for each of the four sites of interest are shown in Figure 1. These density representations help to characterize the developmental patterns present in the data by showing the differences in frequencies across the entire data set. The results of this density study motivated the use of PCA to capture the relation between the sea state variables of interest.

\subsection{Application of PCA to the current problem}

Principal component analysis was used to remove the correlation between energy period and significant wave height for each dataset. The application of PCA generates two new variables that will be called component one $\left(C_{1}\right)$ and component two $\left(C_{2}\right)$, where $C_{1}$ represents the component with the largest variance. The application of PCA to the original $H_{s}$ and $T_{e}$ data yields a coefficient matrix defining a linear combination that allows for rotation into the principal component space. The rotational axes defined by this linear combination are shown in Figure 2. The general form of this coefficient matrix is shown below:

$$
\mathbf{V}=\left[\begin{array}{ll}
v_{1,1} & v_{1,2} \\
v_{2,1} & v_{2,2}
\end{array}\right]
$$


where $v_{2,2}=-v_{1,1}$ and $v_{1,2}=v_{2,1}$. For $\mathrm{i}=1, \ldots, \mathrm{n}$ where $\mathrm{n}$ is the number of observations in the data set under consideration, the equations for each component based on the application of the coefficient matrix above for each point $p_{i}=\left(H_{s_{i}}, T_{e_{i}}\right)$ are shown in Equations 2 and 3:

$$
\mathbf{U}=\left[\begin{array}{cc}
H_{s_{1}} & T_{e_{1}} \\
\vdots & \vdots \\
H_{s_{n}} & T_{e_{n}}
\end{array}\right]
$$

$$
\mathbf{C}=\mathbf{U V}=\left[\begin{array}{cc}
C_{1_{1}}=H_{s_{1}} v_{1,1}+T_{e_{1}} v_{2,1} & C_{2_{1}}=H_{s_{1}} v_{1,2}+T_{e_{1}} v_{2,2} \\
\vdots & \vdots \\
C_{1_{n}}=H_{s_{n}} v_{1,1}+T_{e_{n}} v_{2,1} & C_{2_{n}}=H_{s_{n}} v_{1,2}+T_{e_{n}} v_{2,2}
\end{array}\right]
$$

In order to fulfill the requirements for subsequent elements of the extreme event analysis (i.e., fitting probability distributions to the data), the rotated components must also be shifted upwards along the y axis to ensure that they are greater than zero. This is achieved by applying a shift $s$ where $s=\left|\min \left(C_{2}\right)\right|+0.1$. The final equations for the components defined by a single original data point $p_{i}=\left(H_{s_{i}}, T_{e_{i}}\right)$ are then given by:

$$
\begin{gathered}
C_{1_{i}}=H_{s_{i}} v_{1,1}+T_{e_{i}} v_{2,1} \\
C_{2_{i}}=H_{s_{i}} v_{1,2}+T_{e_{i}} v_{2,2}+s
\end{gathered}
$$

The components defined by these equations are used throughout the remainder of the extreme event analysis until they are transformed back into the original space defined by the sea state variables $H_{s}$ and $T_{e}$. PCA is a bijective transformation, meaning that there is only one way to transform back into the original input space and that the inverse transformation is also a simple linear combination. Given a point $p_{i}=\left(C_{1_{i}}, C_{2_{i}}\right)$ in the principal component space, the transformation back to the corresponding point in the original input space 
defined by $H_{s}$ and $T_{e}$ is defined by:

$$
\begin{aligned}
H_{s_{i}} & =\frac{C_{1_{i}} v_{1,1}+\left(C_{2_{i}}-s\right) v_{1,2}}{v_{1,1}^{2}+v_{1,2}^{2}} \\
T_{e_{i}} & =\frac{C_{1_{i}} v_{1,2}-\left(C_{2_{i}}-s\right) v_{1,1}}{v_{1,1}^{2}+v_{1,2}^{2}}
\end{aligned}
$$

These equations are used to transform the calculated values for the extreme event contour into the input space following the application of the I-FORM described in the remainder of this paper.

\subsection{Discussion}

There is a definite correlation between energy period and significant wave height, as shown in Figures 1 and 2. Dissociating these two variables and treating them independently, as is done in the traditional approach to extreme sea state characterization, underestimates the inherent dependency that these variables share. The principal components in the new basis will be uncorrelated and, thus, the application of PCA will capture some of the dependency between the initial variables $H_{s}$ and $T_{e}$. Correlation is, however, only one aspect of the dependency between these two variables. Therefore, some dependency that is not captured by PCA remains. This dependency is expressed in the nonlinear development of data density over time, as shown in Figure 1. This remaining dependency will be taken into account in the distribution fitting portion of the I-FORM application, and will be discussed in Section 3.2.

\section{Distribution and parameter model fitting}

Following the rotation of the input variables into the principal component space using PCA, distribution and parameter models must be developed for the subsequent component variables so that the I-FORM can be applied to develop environmental contours describing 
extreme sea states. These distribution and parameter models are developed such that they capture some of the remaining dependency between $C_{1}$ and $C_{2}$ that was not taken into account through the application of PCA.

\subsection{Distribution fitting for Component 1}

Following the rotation of the dataset into the principal component space, the cumulative distribution function (CDF) of $C_{1}$ is fitted with an inverse Gaussian distribution using a maximum likelihood estimation technique applied in Matlab. This component was chosen for the initial fitting because it has the largest variance, as can be seen in Figure 2. The inverse Gaussian distribution was used both because it provides a good fit of the CDF shape of $C_{1}$ observed in the datasets under study and because of the simplicity of its defining parameters in terms of interpretation. The equation for the CDF of the inverse Gaussian distribution is given by:

$$
F(x)=\Phi\left(\sqrt{\frac{\lambda}{x}}\left(\frac{x}{\mu}-1\right)\right)+\exp \left(\frac{2 \lambda}{\mu}\right) \Phi\left(-\sqrt{\frac{\lambda}{x}}\left(\frac{x}{\mu}+1\right)\right)
$$

where $\Phi$ represents the CDF of the standard normal distribution and $\mu$ and $\lambda$ can be roughly interpreted as representing the location and scale of the inverse Gaussian distribution (Folks and Chhikara, 1978). The results of the inverse Gaussian fitting of the CDF of $C_{1}$ for all four study sites are shown in Figure 3. This figure also shows the distribution fitting at each site at the highest quantiles. This area is the most important in terms of goodness of fit for the application of the I-FORM to follow because this area will be evaluated during the extrapolation of these distributions as the final environmental contour is constructed. While the inverse Gaussian distribution fits the CDF of $C_{1}$ for three of the study sites well, the fitting is not as good as expected for NDBC 51202, especially at the highest quantiles. This fitting issue predicts a smaller value for $C_{1}$ than is observed at a given quantile for this site, resulting in an underestimation of extreme events by the final 
environmental contour, as seen in Figure 11(c). The selection of a more generic distribution or a mixed distribution to fit the variation in behavior for $C_{1}$ over the selection of study sites might contribute to the creation of more accurate extreme sea state contours across a wider variety of study sites and remains as an area of possible future improvement.

\subsection{Binning Component 2 according to Component 1}

The values of $C_{2}$ are sorted according to their corresponding $C_{1}$ values and split into bins containing 250 observations up to the last group, which contains all remaining points. This number was chosen based on the original sample sizes of the study sites under consideration because it is large enough to generate a good fit and small enough so that the influence of $C_{1}$ on $C_{2}$ can be captured with a fine enough discretization. The sensitivity of the root mean square error of the distribution parameter fitting models, described in the following section, and the final environmental contour to the size of the bins of $C_{2}$ is discussed in detail in Section 3.4. This binning scheme and the distribution and parameter fittings that follow help to capture some of the remaining dependency between $C_{1}$ and $C_{2}$. The CDFs for all bins of $C_{2}$ are shown for all study sites in Figure 4. The CDF of $C_{2}$ for each bin must be fitted with a probability distribution in order for the I-FORM to be applied. The parameters of the $C_{2}$ distributions are fit as functions of the representative value of $C_{1}$ for each bin in order to represent the dependency between $C_{1}$ and $C_{2}$ that has not yet been captured. Based on the shape of the CDFs for each bin of $C_{2}$, a normal distribution was chosen to fit each $\mathrm{CDF}$ of $C_{2}$. This fit seems appropriate considering the symmetry of the data, shown in Figure 4. The parameters that define the normal distribution are the mean, $\mu$, and standard deviation, $\sigma$. The equation for the CDF of the normal distribution is given by:

$$
\Phi\left(\frac{x-\mu}{\sigma}\right)=\frac{1}{2}\left[1+\operatorname{erf}\left(\frac{x-\mu}{\sigma \sqrt{2}}\right)\right]
$$


where erf represents the error function (Johnson et al., 1995). The CDFs for selected bins of $C_{2}$ along with their corresponding normal distribution fits for all study sites are shown in Figure 5. These normal distribution fits were generated using a maximum likelihood estimation technique for $\mu$ and the square root of the unbiased estimate of the variance for $\sigma$

\subsection{Fitting functions for Component 2 distribution parameters $\mu$ and $\sigma$}

The sets of $\mu$ and $\sigma$ values that are created following the fitting of a normal distribution to the CDFs of $C_{2}$ are shown as functions of the mean value for $C_{1}$ for each bin at each study site in Figure 6. Based on the trends observed in the data, it was determined that a simple linear approximation could be used to fit $\mu$ as a function of $C_{1}$ and a constrained quadratic approximation could be used to fit $\sigma$. The fitting functions for $\mu$ and $\sigma$ are as follows:

$$
f_{\mu}\left(C_{1}\right)=m_{1} C_{1}+m_{2}
$$

$$
f_{\sigma}\left(C_{1}\right)=s_{1} C_{1}^{2}+s_{2} C_{1}+s_{3}
$$

In order to ensure that the quadratic fit for $\sigma$ remains positive for small values of the mean of $C_{1}$, the least squares fit for this parameter is subjected to the following constraints:

$$
\begin{gathered}
s_{3} \geq 0 \\
s_{3}-\frac{s_{2}^{2}}{4 s_{1}} \geq 0
\end{gathered}
$$

These constraints ensure that both the $y$-intercept and the minimum of this quadratic model remain greater than or equal to zero. The fitting function for $\mu$ was fit to the data using a basic linear least squares technique. The $\sigma$ function was fit using a penalty function that applies the constraints given above to a least squares objective function evaluated using a direct search, derivative-free method applied in Matlab. The final models for $\mu$ and $\sigma$ at 
each study site for the binning scheme described above are shown in Figure 6 along with the RMSE for each fit.

Although these models do not perfectly represent the variations present in the data, they allow for smooth extrapolations that create more practically applicable environmental contours. This is especially true when the data appears to be unstructured and may contain multimodal distributions, as is seen in Figure 1(c).

\subsection{Bin size sensitivity study}

A sensitivity study was conducted to determine the impact of the size of the $C_{2}$ bins on both the root mean square error (RMSE) of the fitting models for $\mu$ and $\sigma$ and the final environmental contour at one site (NDBC 46212). Bin sizes were tested at intervals of 25 data points from a bin size of 50 points up to 10000 points. For each of these bins, the RMSEs for the fitting models of $\mu$ and $\sigma$ are shown in Figures 7 and 8, respectively.

In order to stabilize the response of the parameter fitting functions with respect to the size of each bin, the values for $\mu$ and $\sigma$ for the last bin were not included in the calculation of each fitting model. This ensures that the trends found in the majority of each parameter dataset, rather than possible extreme values created by a small number of data points in the last bin, are used to generate the coefficients for each parameter model. The responses of the RMSE for the models of $\mu$ and $\sigma$ when the parameters of the last bin of $C_{2}$ are included in the fitting calculation are shown in blue in Figures 7 and 8 and are compared to the same responses when the last bin is not included, which are shown in green. The number of points in the final bin for each fixed bin size is also shown in these figures on a secondary $y$-axis. This shows that peaks in the RMSE for both $\mu$ and $\sigma$ when the final bin is included in the fitting calculation often correspond to points at which there are very few points in the final bin. When these points are no longer included, the RMSE stabilizes for the $\mu$ fitting function and reaches a value between 0.025 and 0.05 (Figure 7). The RMSE also stabilizes 
for the $\sigma$ fitting function up to a bin size of approximately 5500 data points when the final bin is not included in the fitting calculation(Figure 8). After this point, some peaks in the RMSE still remain. These peaks may correspond to numerical instabilities in the fitting algorithm caused by the large number of points included in each bin, which create a small number of points with little continuity for the $\sigma$ function to fit. This provides a justification for choosing a bin size (e.g., 250) that is large enough to contain a representative number of points for the normal distribution fit but small enough that the parameter values for each parameter modeling function are continuous as a function of the mean value of $C_{1}$ for each bin, allowing for the generation of a close-fitting parameter model.

The final contours for each modeled bin size up to a bin size of 5000 points are shown in Figure 9. These contours show very little variation with a maximum significant wave height difference at a single contour point of 0.26 meters and maximum energy period difference of 0.08 seconds. This shows that the resulting contour will not be significantly different than the same contour for a slightly different bin size when the fitting functions for $\mu$ and $\sigma$ have stable, relatively low RMSEs.

\section{I-FORM Application}

Following the creation of an inverse Gaussian distribution fit for component one and the development of models fitting the parameters of the normal distributions of the bins of $C_{2}$ as a function of the mean value of $C_{1}$ for each bin, the inverse first-order reliability method (I-FORM) can be applied to construct an environmental contour for a given return period.

\subsection{Contour creation}

In the standard FORM approach, a threshold value is considered and its likelihood is estimated in the standard normal space. The inverse FORM approach, described by Haver 
(2009), starts with an exceedance probability (i.e., a return period of 100 years) that defines the radius $\beta$ of an isoline in the standard normal space. Equation 14 shows the calculation of the probability for a given return period $\left(t_{r}\right)$, given in years, based on the measurement interval $\left(t_{s}\right)$, given in hours. The subsequent calculation of $\beta$ is shown in Equation 15:

$$
\begin{gathered}
p=\frac{1}{365 * \frac{24}{t_{s}} * t_{r}} \\
\beta=\Phi^{-1}(p)=\sqrt{2} \operatorname{erf}^{-1}(2 p-1)
\end{gathered}
$$

where $\Phi^{-1}$ is the inverse CDF of the standard normal distribution. This isoline is then transposed into the original uncertain input space in order to evaluate the range of extreme values. Numerically, a discretization is used on the angle $\theta$ over the isoline to generate a set of points $U_{j}=\left(u_{1_{j}}, u_{2_{j}}\right)$, represented as the following parametric function:

$$
\begin{aligned}
& u_{1_{j}}=\beta \cos \left(\theta_{j}\right) \\
& u_{2_{j}}=\beta \sin \left(\theta_{j}\right)
\end{aligned} \text { where } \theta_{j}=\frac{2 \pi j}{k}, j=1, \ldots, k
$$

For each value of $j$, the quantile position $Q_{j}=\left(q_{1_{j}}, q_{2_{j}}\right)$ of the chosen probability of likelihood is calculated in both directions using the inverse of the standard normal cdf:

$$
\begin{aligned}
& q_{1_{j}}=\Phi^{-1}\left(u_{1_{j}}\right) \\
& q_{2_{j}}=\Phi^{-1}\left(u_{2_{j}}\right)
\end{aligned}
$$

where $\Phi^{-1}$ is the inverse of the equation for the standard normal CDF. The resulting quantile $q_{1_{j}}$ is then used to evaluate the inverse of the inverse Gaussian CDF to obtain a value for $C_{1}$ :

$$
C_{1_{j}}=F^{-1}\left(q_{1_{j}}\right)
$$

where $F$ is the equation for the inverse Gaussian distribution with parameters that were fit to the distribution of $C_{2}$ obtained from the original input data, described in Section 3.1. 
This value of $C_{1}$ is used to evaluate the normal parameter models in order to obtain a particular distribution of $C_{2}$ :

$$
\begin{aligned}
\mu_{j} & =m_{1} C_{1_{j}}+m_{2} \\
\sigma_{j} & =s_{1} C_{1_{j}}{ }^{2}+s_{2} C_{1_{j}}+s_{3}
\end{aligned}
$$

The quantile position $q_{2_{j}}$ is used to evaluate the inverse of the particular normal CDF defined using the parameters calculated above in order to obtain a value for $C_{2}$ at this point:

$$
C_{2_{j}}=\sigma_{j} \sqrt{2}\left[\operatorname{erf}^{-1}\left(2 q_{2_{j}}-1\right)\right]+\mu_{j}
$$

This process is performed for each value along the discretization of the isoline in the standard normal space to create a complete environmental contour in the principal component space. The values of each point on this contour must then be transformed from the principal component space into the original sample space defined by the variables $H_{s}$ and $T_{e}$ using Equations 6 and 7, respectively. The extreme sea state contours resulting from this methodology at the four study sites under consideration are shown in Figure 10.

\subsection{Results}

The environmental contours generated using the modified I-FORM at each of the four study sites are shown with the corresponding contours created using the traditional methodology (e.g., Berg (2011)) in Figure 11. The contours that are developed under the new methodology are displayed along with a representation of the density of each data set under consideration, calculated as described in Section 2.1. This representation helps to emphasize the importance of considering the conjoint influence of energy period and significant wave height in order to develop extreme sea state contours that reflect the density patterns in each dataset. The contours created using the new methodology appear to follow the shape of trends present within the data, a great improvement on the contours 
created using the traditional methodology. This allows for coverage in the area of the input space in which both energy period and significant wave height are high, a possible area of importance for survivability analysis of marine structures. The new shape of these contours is similar to the shape of environmental contours obtained by Silva-González et al. (2013), which also provide a better coverage of the input space of interest than the contours obtained using the traditional methodology.

At the most basic level, the extreme sea state contours created using the new methodology show a much better coverage of the data for the given period of record at each of the study sites under consideration. It is expected that, given a period of record on the order of tens of years, the extreme contour for a return period on the order of hundreds of years should include all of the observed data. This is true for all of the study sites with the exception of NDBC 51202. At this site, the complex relations between $H_{s}$ and $T_{e}$ are not entirely captured by the application of both principal component analysis and the distribution and parameter fitting. Investigation into the patterns that are visible in this dataset, shown in Figure 1(c), found that the long fingers of observations stretching beyond the majority of the points belong to individual storm events. These storm events are consistent with the large annual winter storms in the North Pacific that have been found to have a strong impact on significant wave height in general (Gulev and Grigorieva, 2006) and on significant wave height in Hawaii in particular (Stopa et al., 2011). The extreme event contour at this site is perturbed by these storms because they are relatively too infrequent to be captured by the methodologies proposed in this paper. In addition, the distribution fitting functions described in Section 3 do not seem as appropriate for the CDFs of Component 1 and Component 2 at this site when compared to the other three sites, as shown in Figures 3 and 5. This suggests that the distributions of these variables, and the corresponding distributions of significant wave height and energy period, do not follow the same patterns as those for the variables at the other three sites. This difference may stem from the 
variations in oceans hydrodynamics amongst continental and island landforms. The wave dynamics at NDBC 51202 are subject to phenomena that propagate wave energy around the island, causing waves to approach from varying directions (Bascom, 1980). Furthermore, the topography of the Hawaiian Islands has been found to modify tradewind flow, causing local wind acceleration that may also increase the wave energy found at this location (Stopa et al., 2011). The combination of all of these effects, which are unique to the Hawaiian islands, helps to explain the different patterns found in the data from this location that contribute to inconsistencies in the final environmental contour generated for this site. Possible improvements to the environmental contour at this site could be made by (a) collecting more data, (b) using surrogate models, or (c) including data from a similar site. The improvement of the calculated contour at sites with these types of sea state patterns remains as an area of future work beyond the scope of the current analysis.

The maximum significant wave heights for a return period of 100 years predicted using the new methodologies proposed in this work are significantly higher than the maximum values obtained using the traditional methodology implemented as described by Berg (2011) and shown in Figure 11. This particular implementation of the traditional approach uses probability distribution models that do not fit the data as well as they should for extreme value extrapolation. In an effort to find support for the much higher maximum $H_{s}$ values obtained using principal component analysis and the modified I-FORM method, the traditional methodology was implemented with an updated double-Weibull distribution fitting that provided a better fit for the distribution tails of the significant wave height data. The results of this exploration are shown in Figure 12. These show that the improvement of distribution fitting in the implementation of the traditional methodologies that focuses on fitting distribution tails can result in much higher values for the maximum significant wave height, supporting the maximum values found in this work. 


\section{Conclusion}

Overall, this paper presents a significant improvement to the original method of calculating an extreme contour of sea states. The proposed modifications, utilizing principal components, better represent the measured data and, therefore, should contribute to the development of more realistic representations of environmental contours of extreme sea states. This can better prepare developers of marine structures for survivability analysis and can be applied in many areas of interest. The increase in the maximum significant wave height expected to occur for a given return period derived from the new contours developed in this work presents a significant development in the field of marine survivability analysis.

There are several areas that represent possibilities for future enhancements to the methodology developed in this paper. First, the use of principal component analysis to create an orthogonal decomposition of the data such that the values are uncorrelated in each direction only addresses one aspect of the complex relation between energy period and significant wave height, as can be seen in Figure 11(c) and (d). A more complex decomposition taking into account the varying relations among variables across the study sites shown in these representations of density (e.g., curvature) could lead to a better representation of the data and, therefore, a more accurate approximation of the extreme sea state contour for a given return period. However, it is unclear how orthogonality could be preserved under this new paradigm. Second, the selection of a more generic distribution or a mixed distribution to fit the variation in behavior for $C_{1}$ over the selection of study sites along with refinements in the models developed for the normal distribution parameters $\mu$ and $\sigma$ for $C_{2}$ might also contribute to the creation of more accurate extreme sea state contours for all sites. Finally, the use of principal component analysis can easily be extended to include multiple dimensions, with each new set of components ultimately presented as 
a simple linear combination of the original variables. In future work, this could be used to consider additional variables (e.g., wind and water current speed) related to the problem of interest.

\section{Acknowledgments}

This research was made possible by support from the U.S. Department of Energys (DOE) Energy Efficiency and Renewable Energy (EERE) Offices Wind and Water Power Technologies Office. Sandia National Laboratories is a multi-program laboratory managed and operated by Sandia Corporation, a wholly owned subsidiary of Lockheed Martin Corporation, for the U.S. Department of Energys National Nuclear Security Administration under contract DE-AC04-94AL85000.

\section{References}

Bascom, W., 1980. Waves and beaches: The dynamics of the ocean surface. Anchor Press/Doubleday, Garden City, NY.

Berg, J., 2011. Extreme ocean wave conditions for northern California wave energy conversion device. SAND Report SAND2011-9034.

Coe, R. G., Neary, V., Lawson, M., Yu, Y., Weber, J., 2014. Extreme conditions modeling workshop. Technical Report NREL/TP-5000-62305, SAND2014-16384R.

DNV, 2001. Offshore standard- DNV-OSF-201 dynamic risers. Tech. rep., Det Norske Veritas.

DNV, 2002. Recommended practice- DNV-RP-C102 structural design of offshore ships. Tech. rep., Det Norske Veritas. 
DNV, 2008. Offshore service specification- DNV-OSS-312 certification of tidal and wave energy converters. Tech. rep., Det Norske Veritas.

DNV, 2010a. Offshore standard- DNV-OS-E301 position mooring. Tech. rep., Det Norske Veritas.

DNV, 2010b. Recommended practice- DNV-RP-F205 global performance analysis of deepwater floating structures. Tech. rep., Det Norske Veritas.

DNV, 2014. Recommended practice- DNV-RP-C205 environmental conditions and environmental loads. Tech. rep., Det Norske Veritas.

Folks, J., Chhikara, R., 1978. The inverse Gaussian distribution and its statistical application: A review. Journal of the Royal Statistical Society, Series B (Methodological) 40 (3), 263-289.

Forristall, G. Z., Cooper, C., 1997. Design current profiles using empirical orthogonal function (EOF) and inverse FORM methods. Offshore Technology Conference Houston, Texas, 11-21.

Gulev, S., Grigorieva, V., 2006. Variability of the winter wind waves and swell in the North Atlantic and North Pacific as revealed by the voluntary observing ship data. Journal of Climate 19, 116-127.

Haver, S. amd Winterstein, S., 2009. Environmental contour lines: A method for estimating long term extremes by a short term analysis. Transactions, Society of Naval Architects and Marine Engineers 116, 116-127.

Hotelling, H., 1933. Analysis of a complex of statistical variables into principal components. Journal of Educational Psychology 24 (6), 417-441. 
Huseby, A., Vanem, E., Natvig, B., 2013. A new approach to environmental contours for ocean engineering applications based on direct Monte Carlo simulations. Ocean Engineering 60, 124-135.

Jackson, J., 1991. A user's guide to principal components. John Wiley \& Sons, Inc.

Johnson, N., Kotz, S., Balakrishnan, N., 1995. Continuous univariate distributions, 2nd Edition. Vol. 1. J. Wiley, New York.

Lenee-Bluhm, P., Paasch, R., Özkan-Haller, H., 2011. Characterizing the wave energy resource of the US pacific northwest. Renewable Energy 36 (8), 2106-2119.

National Data Buoy Center, 2015a. Station 46022 (LLNR 500)- Eel River- 17NM WSW of Eureka, CA.

URL http: //www . ndbc . noaa . gov/station_page . php? station=46022

National Data Buoy Center, 2015b. Station 46050 (LLNR 641)- Stonewall Bank- 20NM West of Newport, OR.

URL http : //www . ndbc . noaa.gov/station_page . php?station=46050

National Data Buoy Center, 2015c. Station 46212- Humboldt Bay South Spit, CA (128).

URL http: //www . ndbc .noaa.gov/station_page . php?station=46212

National Data Buoy Center, 2015d. Station 51202- Mokapu Point, HI (098).

URL http : //www . ndbc . noaa.gov/station_page . php?station=51202

Nygaard, E., Johannessen, K., 2000. Metocean design criteria for Kvitebjorn. Statoil Report KVB-N-FD-0001.

Pearson, K., 1901. On lines and planes of closest fit to systems of points in space. Philosophical Magazine 2 (11), 559-572. 
Rosenblatt, M., 1952. Remarks on a multivariate transformation. Annals of Mathematical Statistics.

Silva-González, F., Heredia-Zavoni, E., Montes-Iturrizaga, R., 2013. Development of environmental contours using Nataf distribution model. Ocean Engineering 58, 27-34.

Stopa, J., Cheung, K., Chen, Y., 2011. Assessment of wave energy resources in Hawaii. Renewable Energy 36 (2), 554-567.

Vanem, E., Bitner-Gregersen, E., June 2014. Alternative environmental contours for marine structural design: A comparison study. In: Proceedings of the ASME 2014 33rd International Conference on Ocean, Offshore and Arctic Engineering. Vol. 4A: Structures, Safety and Reliability. San Francisco, California.

Winterstein, S., Ude, T., Cornell, C., Bjerager, P., Haver, S., August 1993. Environmental parameters for extreme response: Inverse FORM with omission factors. In: ICOSSAR93. Innsbruck, Austria. 


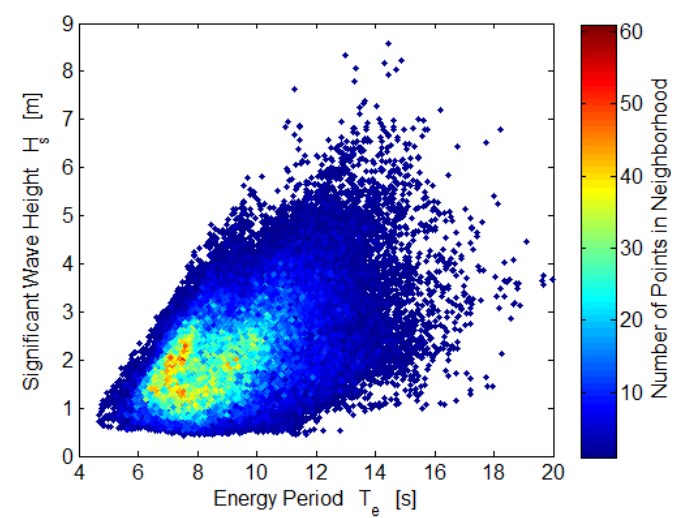

(a)

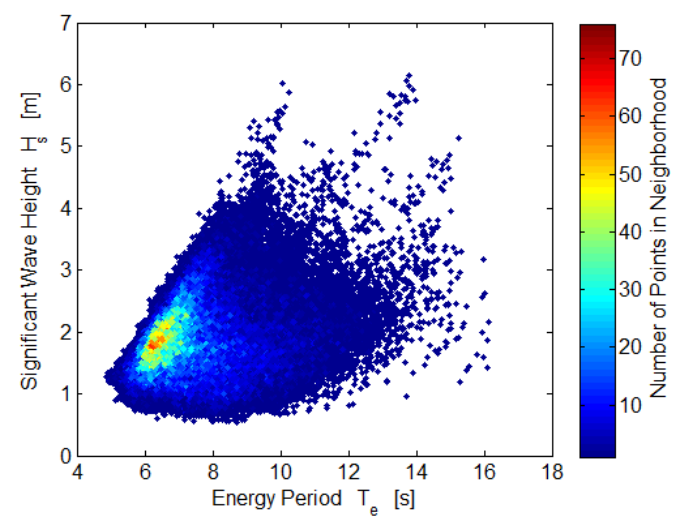

(c)

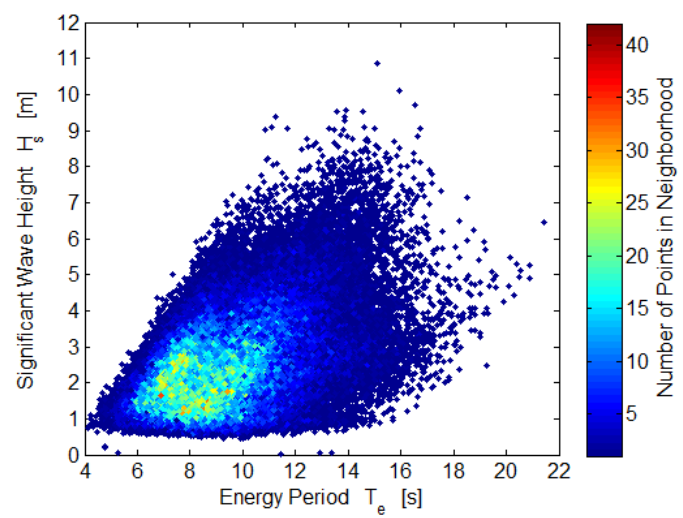

(b)

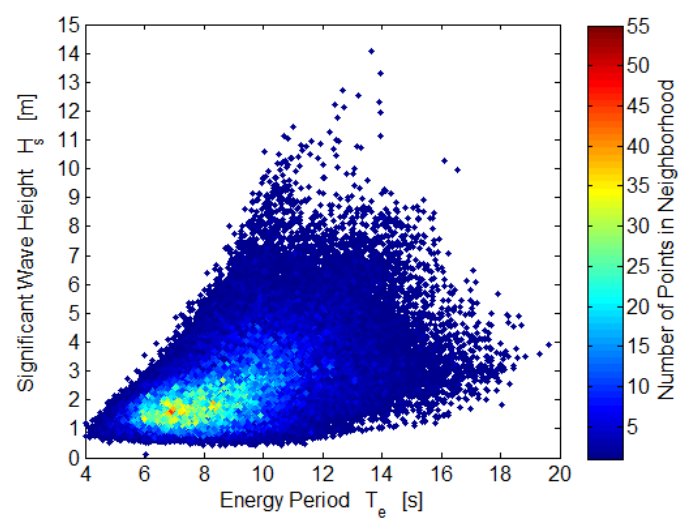

(d)

Figure 1: Representation of data density for four study sites (a) NDBC 46212, (b) NDBC 46022, (c) NDBC 51202, (d) NDBC 46050. 


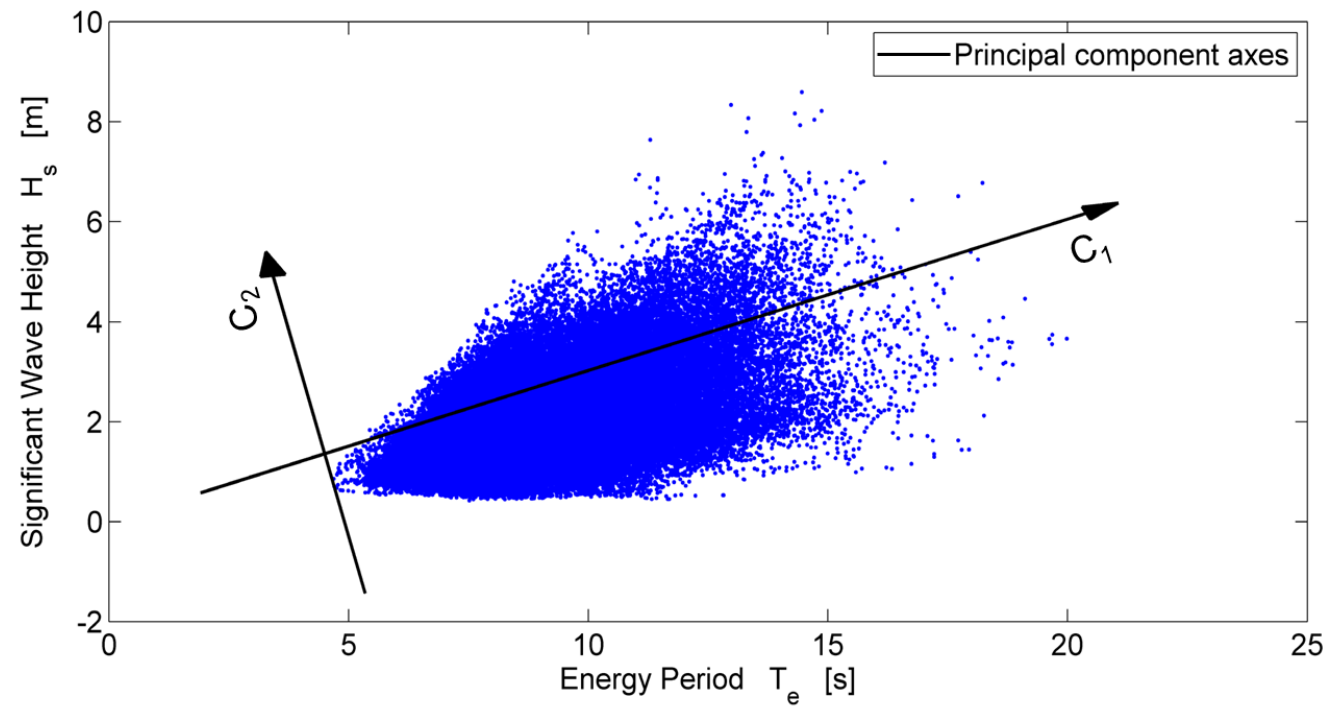

Figure 2: Representation of the axes of the new basis developed using principal component analysis for NDBC 46212. 

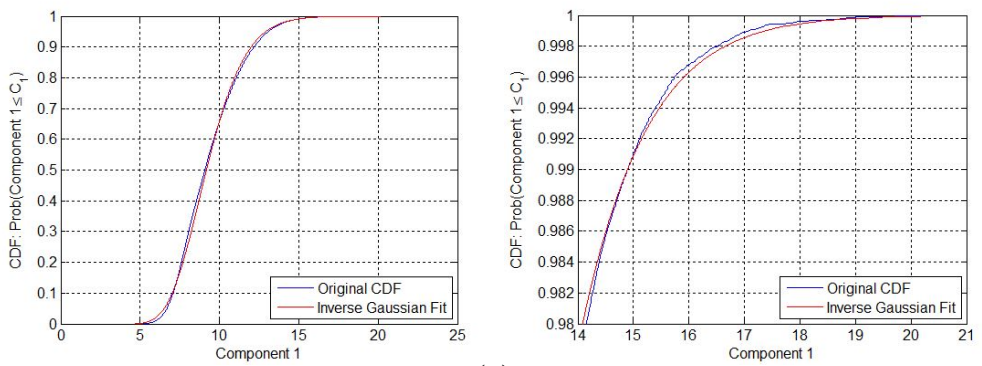

(a)
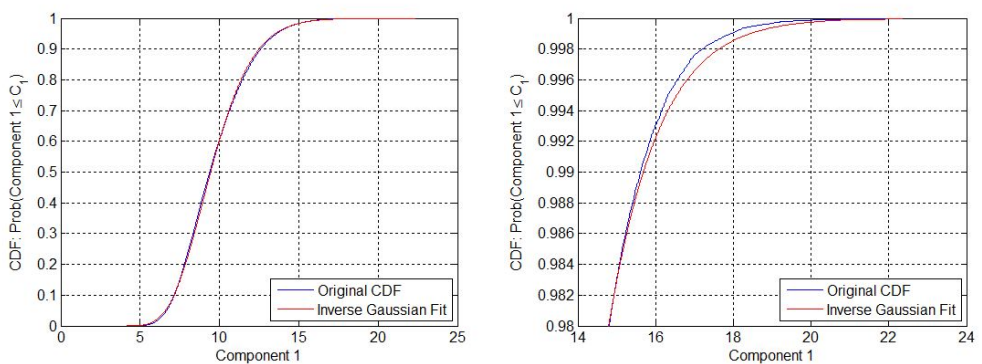

(b)
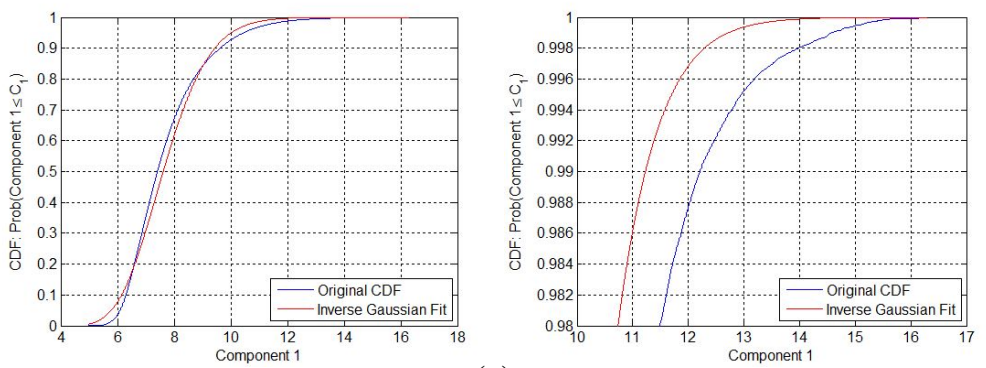

(c)
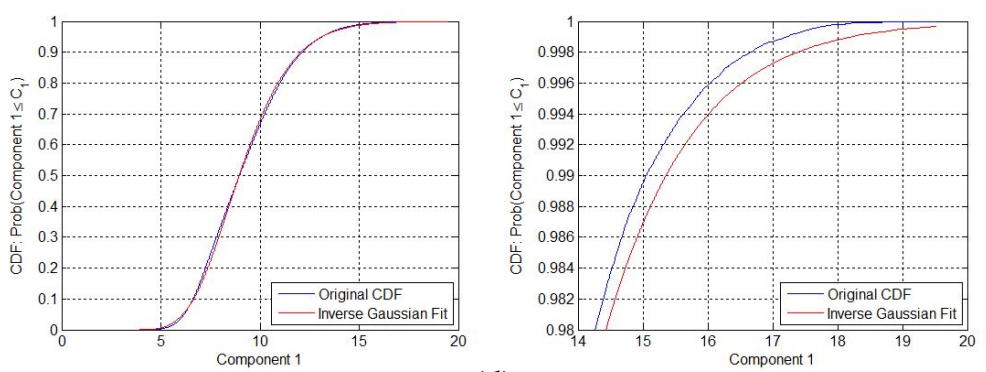

(d)

Figure 3: Component $1 \mathrm{CDF}$ (left) and zoom into highest quantiles (right) for all data (blue) and inverse Gaussian model (red) for (a) NDBC 46212, (b) NDBC 46022, (c) NDBC 51202, (d) NDBC 46050. 


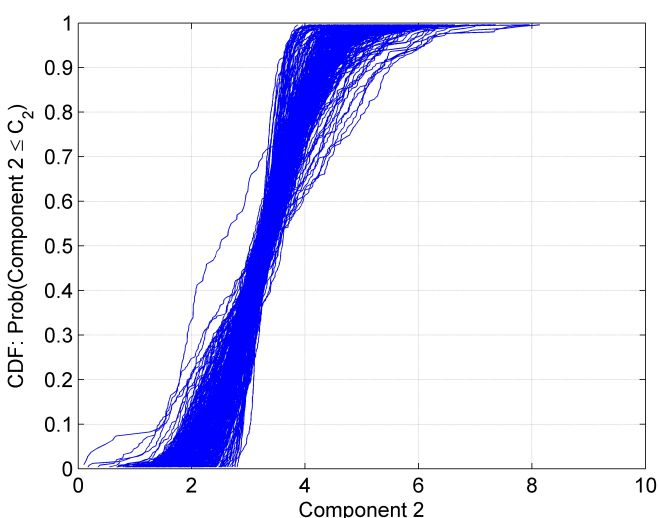

(a)

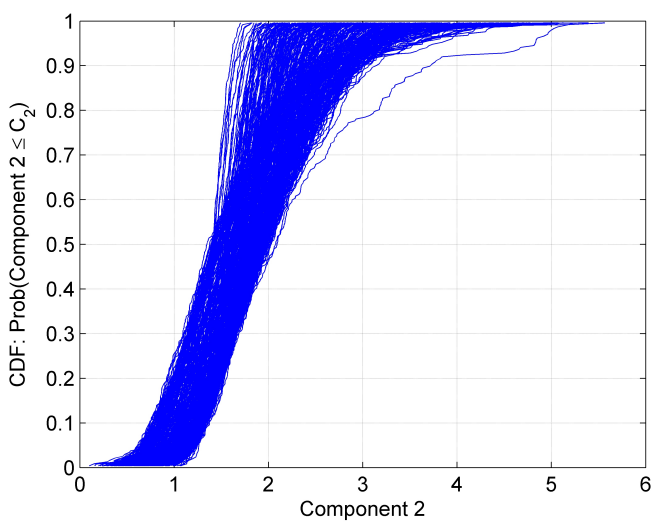

(c)

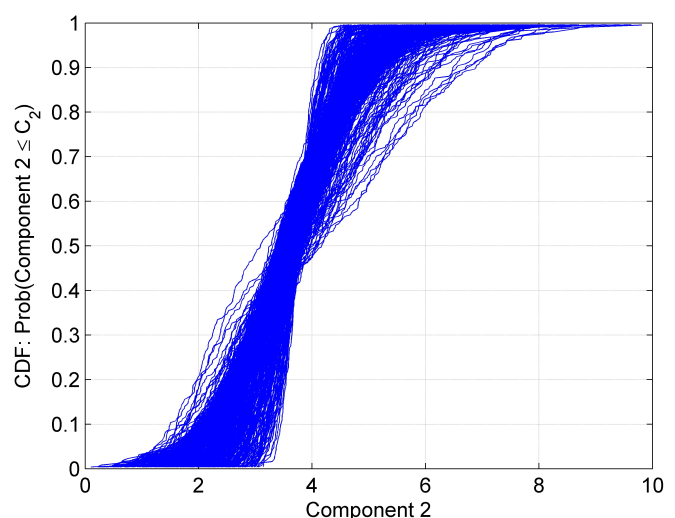

(b)

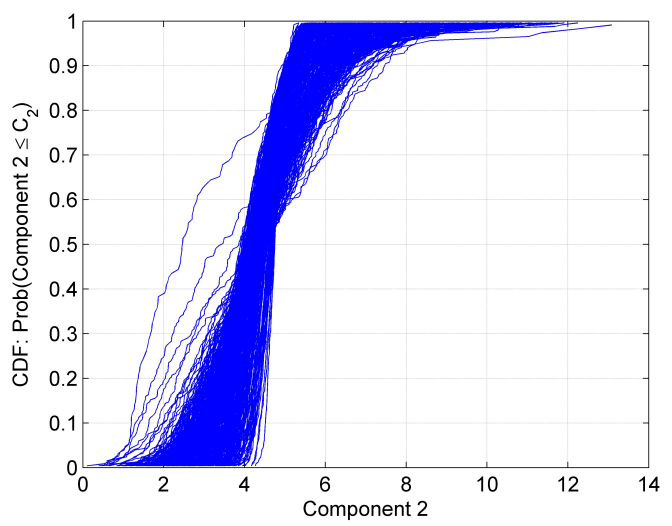

(d)

Figure 4: CDFs for all bins of Component 2 for (a) NDBC 46212, (b) NDBC 46022, (c) NDBC 51202, (d) NDBC 46050. 

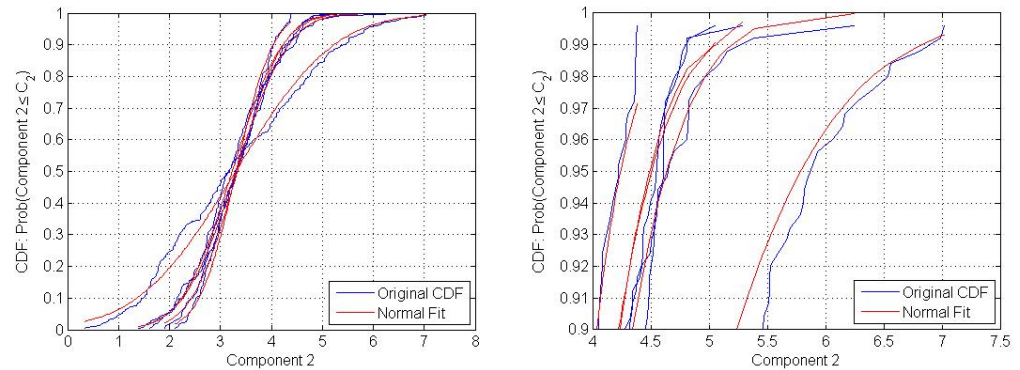

(a)
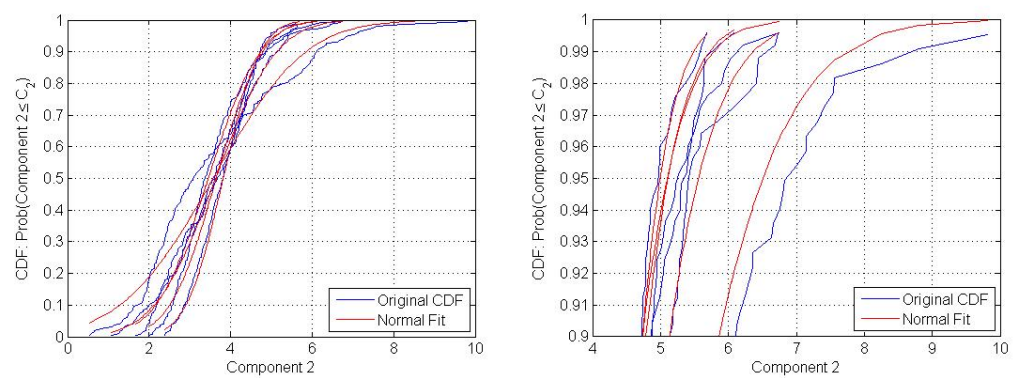

(b)
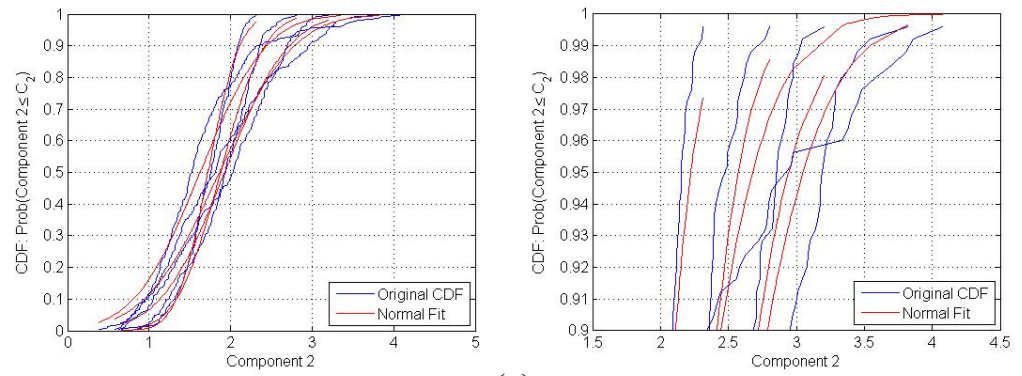

(c)
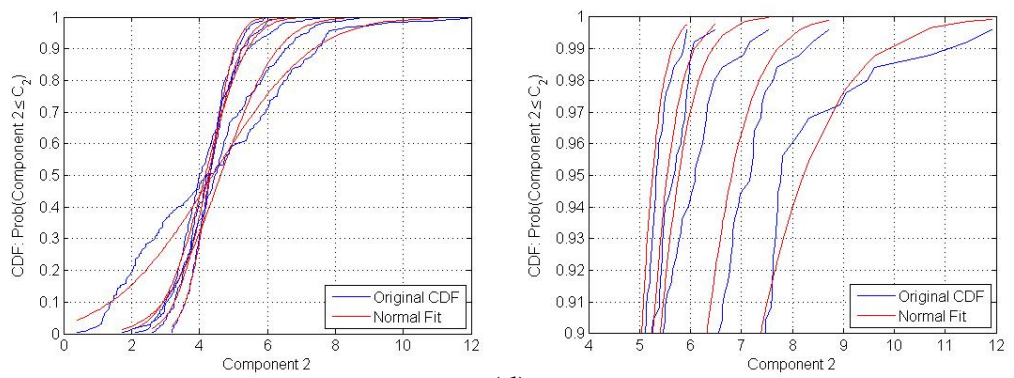

(d)

Figure 5: CDFs for selected bins of Component 2 with normal distribution fits at full scale (left) and zoom into highest quantiles (right) for (a) NDBC 46212, (b) NDBC 46022, (c) NDBC 51202, (d) NDBC 46050. 

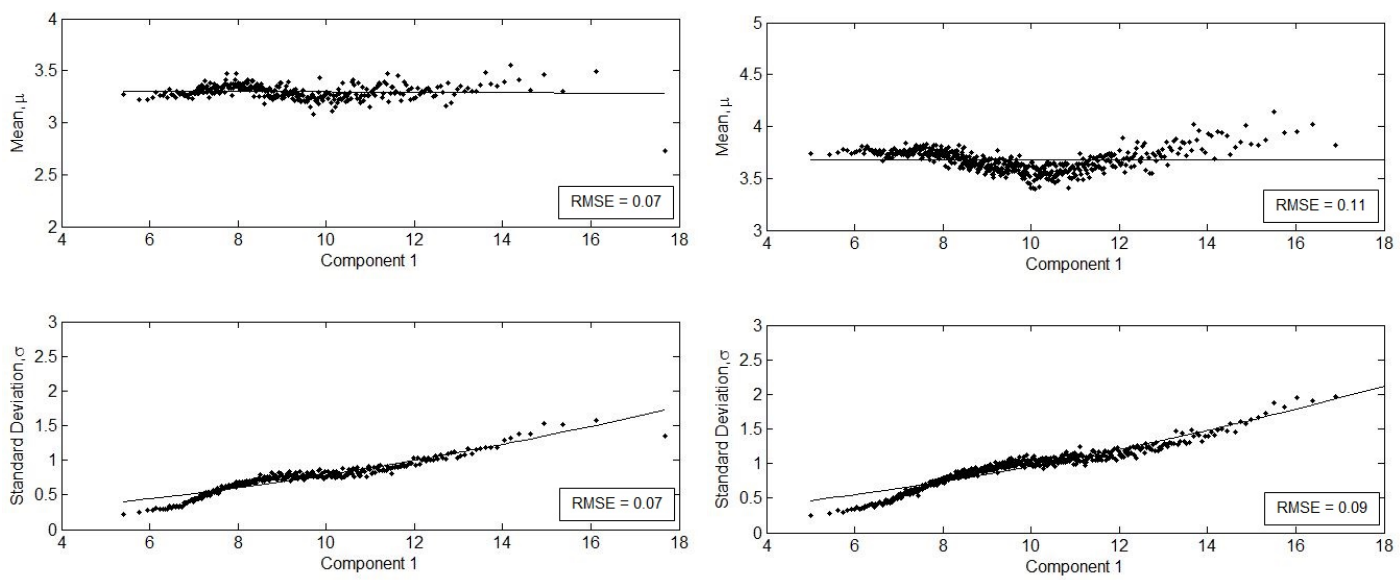

(a)

(b)
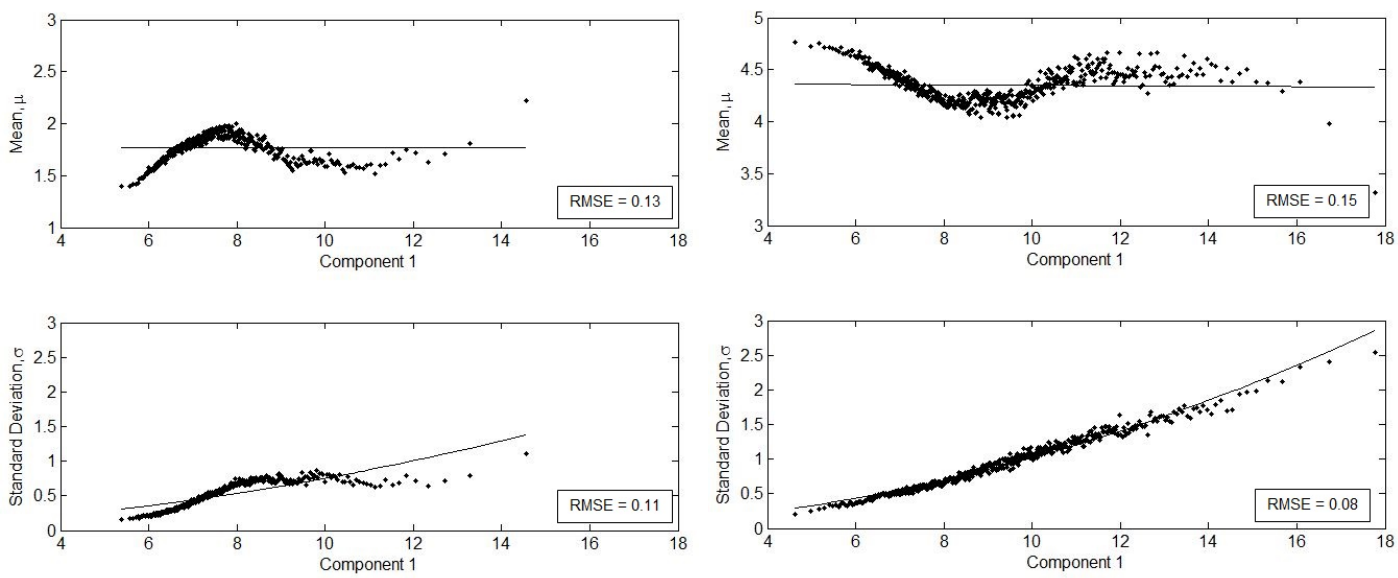

(c)

(d)

Figure 6: Estimates of the Component 2 normal distribution parameters $\mu$ (top) and $\sigma$ (bottom) as a function of Component 1 for each bin for (a) NDBC 46212, (b) NDBC 46022, (c) NDBC 51202, (d) NDBC 46050. 


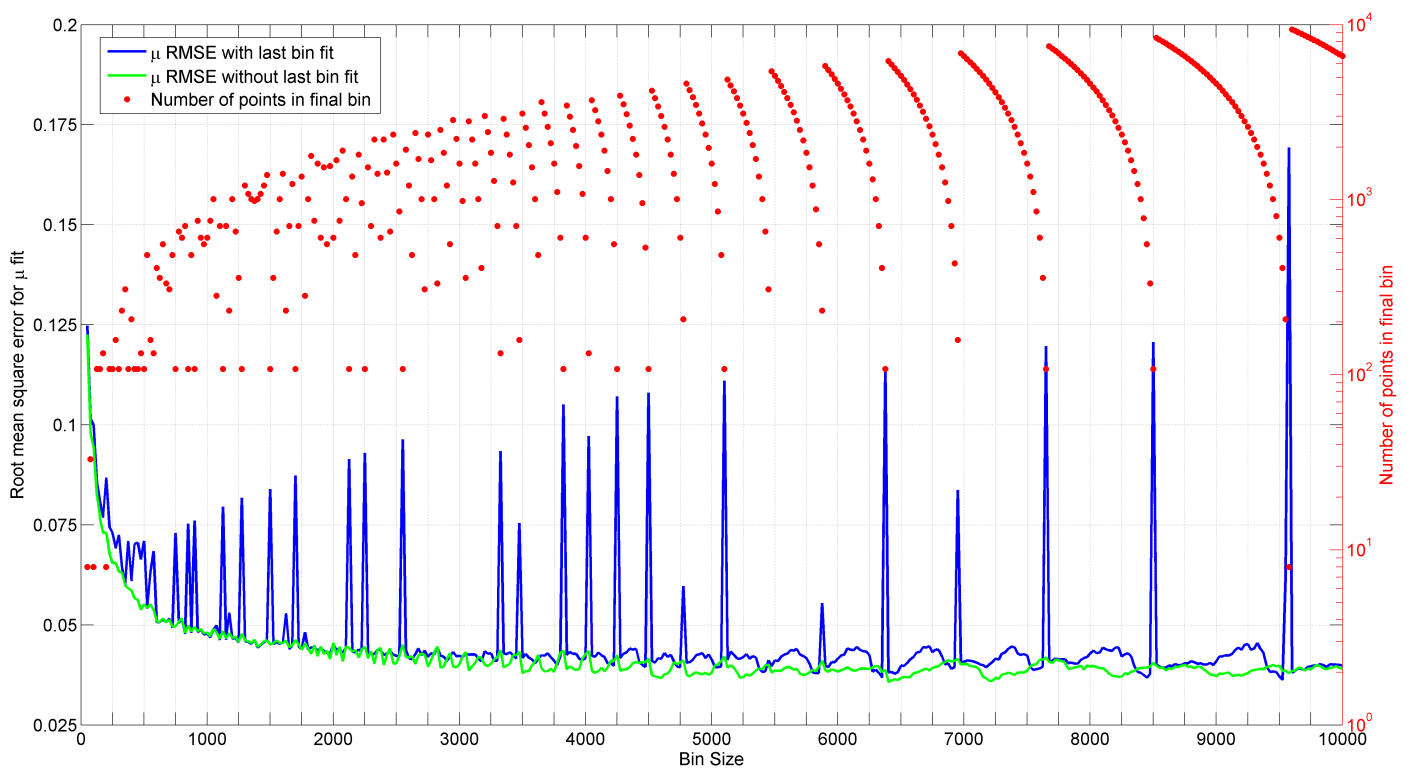

Figure 7: Root mean square error (RMSE) for the $\mu$ fitting function as a function of bin size shown when the last bin is included in the fitting (blue) and when it is not (green) along with the number of points in the final bin as a function of bin size (red) for NDBC 46212. 


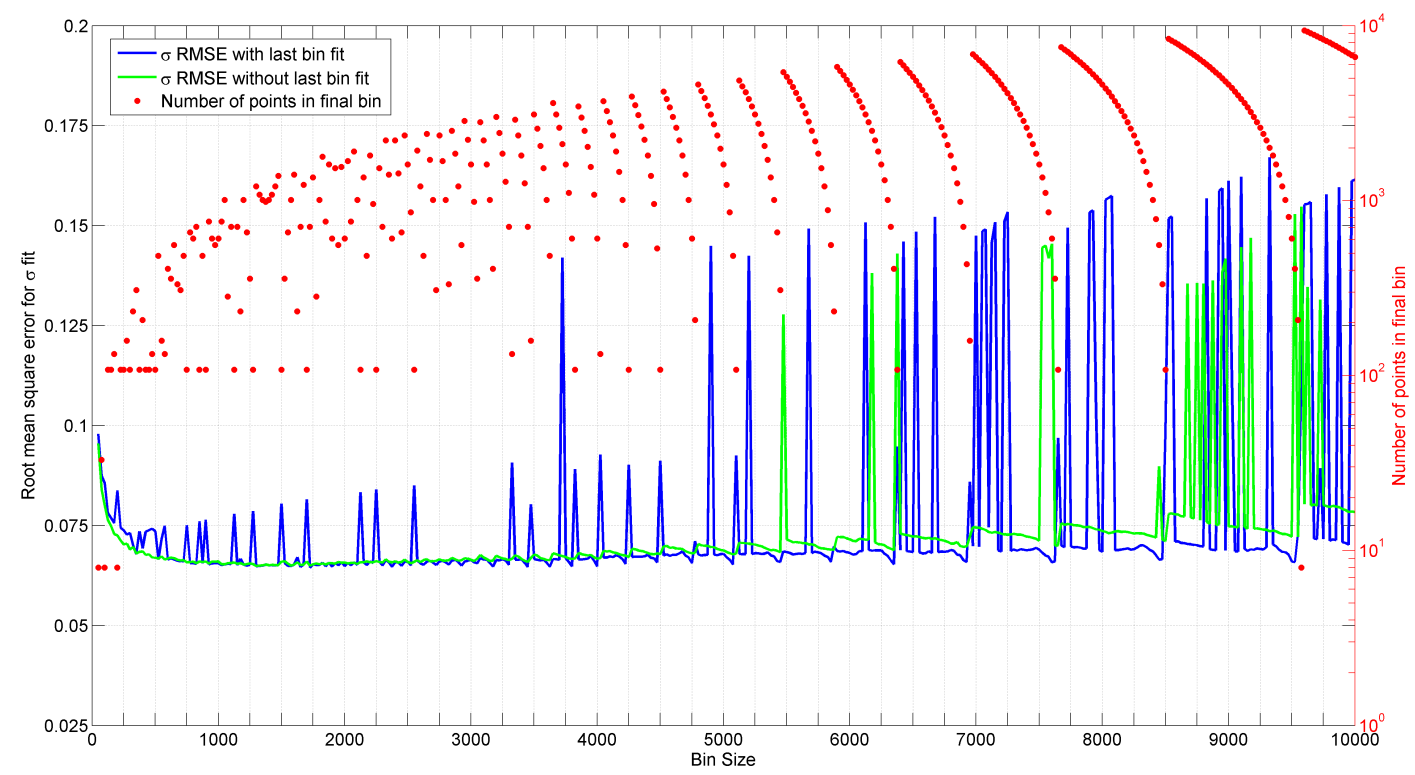

Figure 8: Root mean square error (RMSE) for the $\sigma$ fitting function as a function of bin size shown when the last bin is included in the fitting (blue) and when it is not (green) along with the number of points in the final bin as a function of bin size (red) for NDBC 46212. 


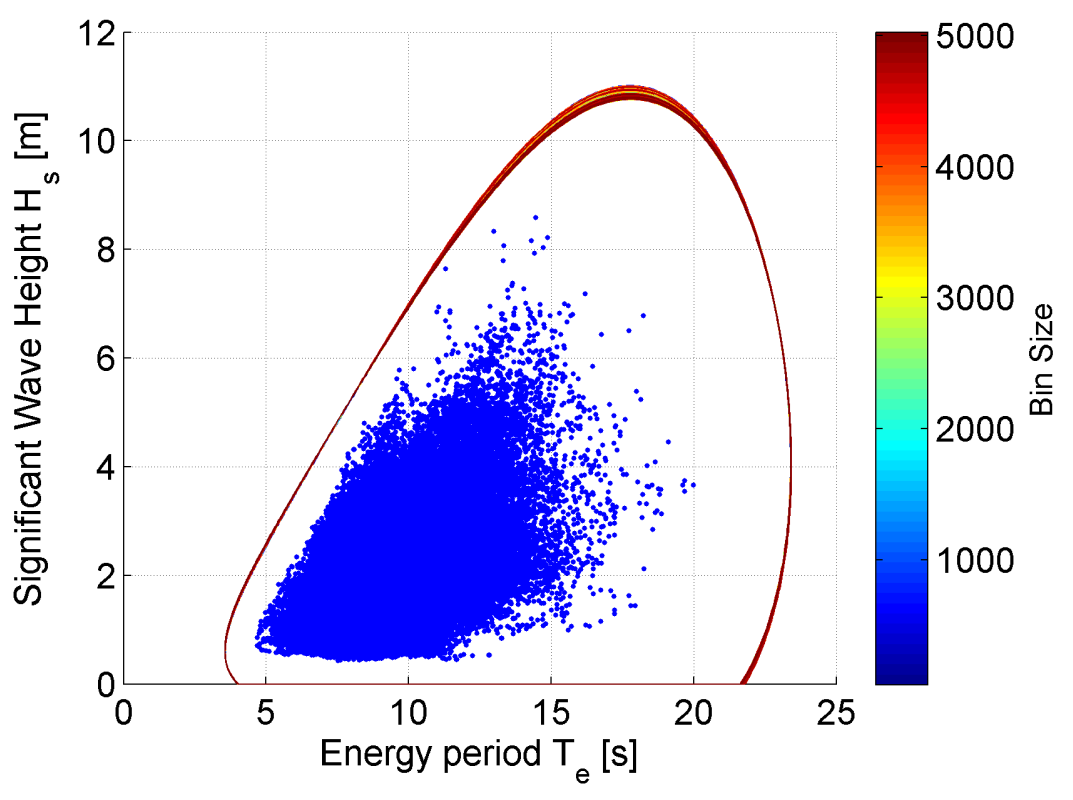

Figure 9: Final 100-year environmental contours for bins modeled at increments of 25 data points from a bin size of 50 points to a bin size of 5000 points for NDBC 46212. 


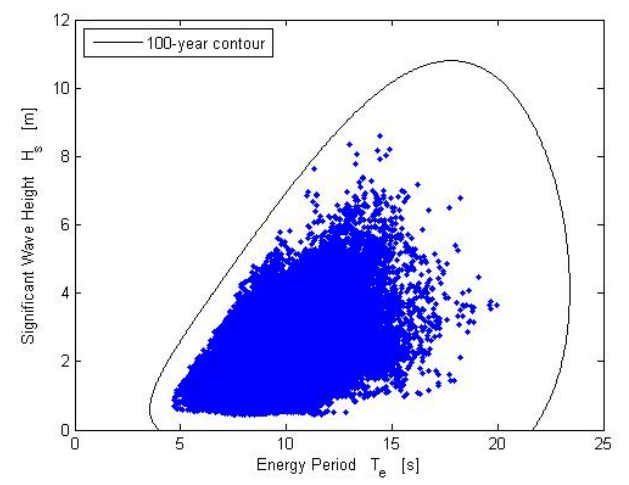

(a)

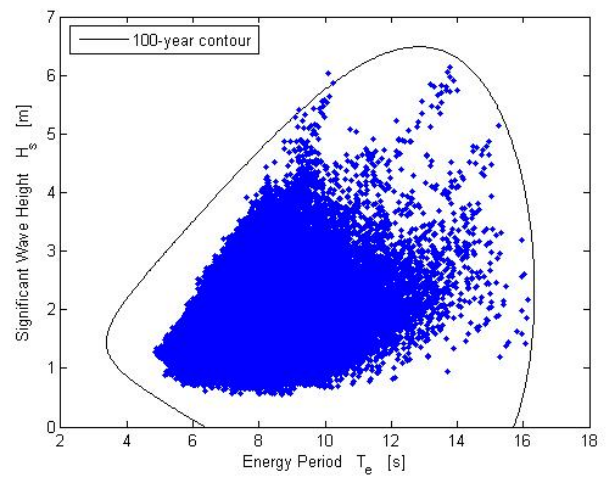

(c)

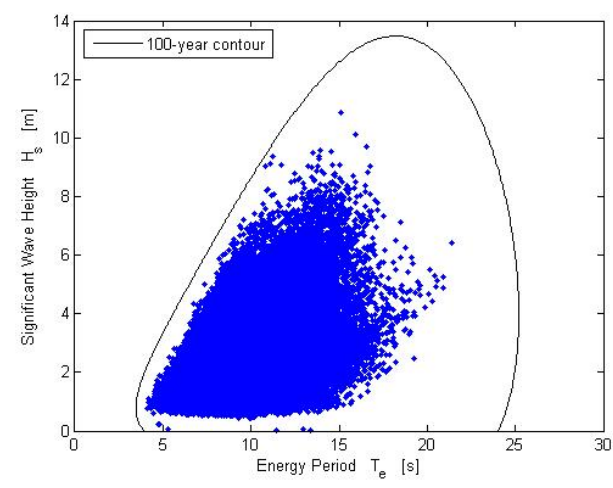

(b)

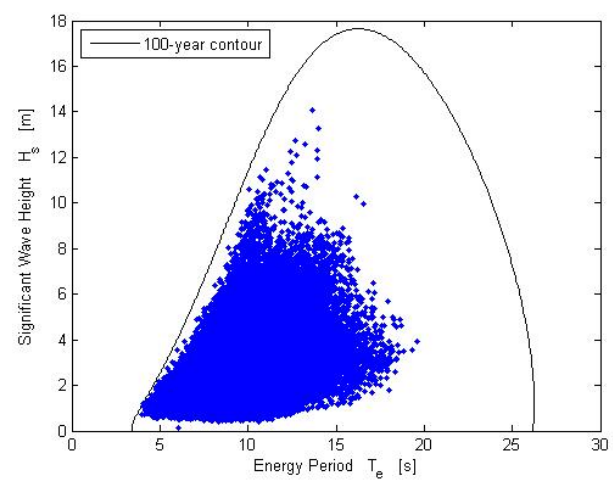

(d)

Figure 10: 100-year extreme sea state contour for (a) NDBC 46212, (b) NDBC 46022, (c) NDBC 51202, (d) NDBC 46050. 


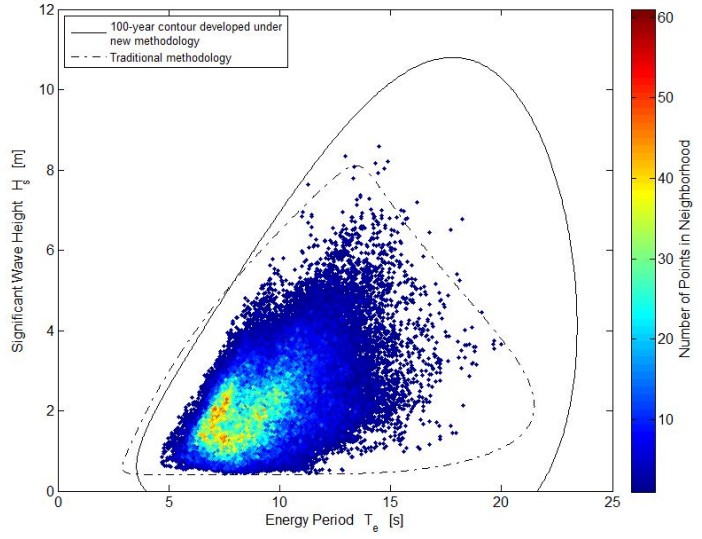

(a)

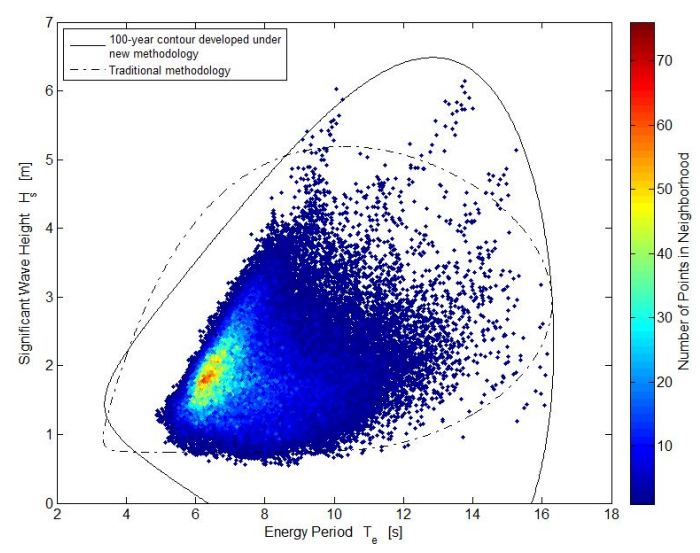

(c)

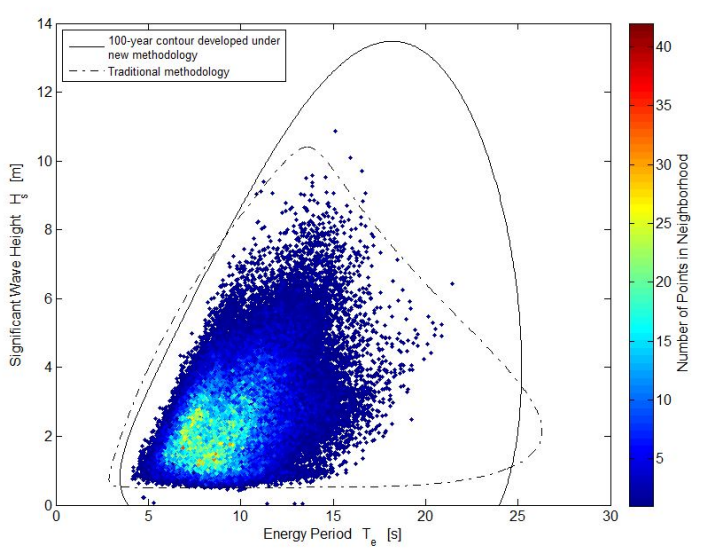

(b)

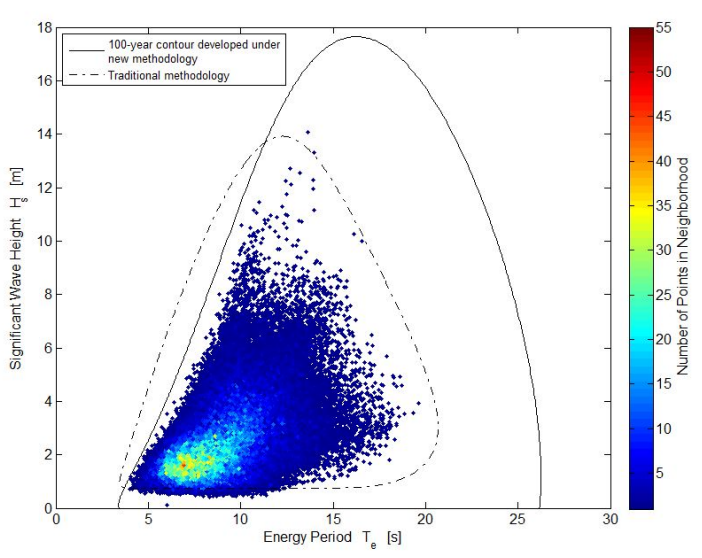

(d)

Figure 11: 100-year extreme sea state contours created by the traditional methodology(dashed line) (Berg, 2011) and the new methodology presented in this paper shown with data density(solid line) for (a) NDBC 46212, (b) NDBC 46022, (c) NDBC 51202, (d) NDBC 46050. 


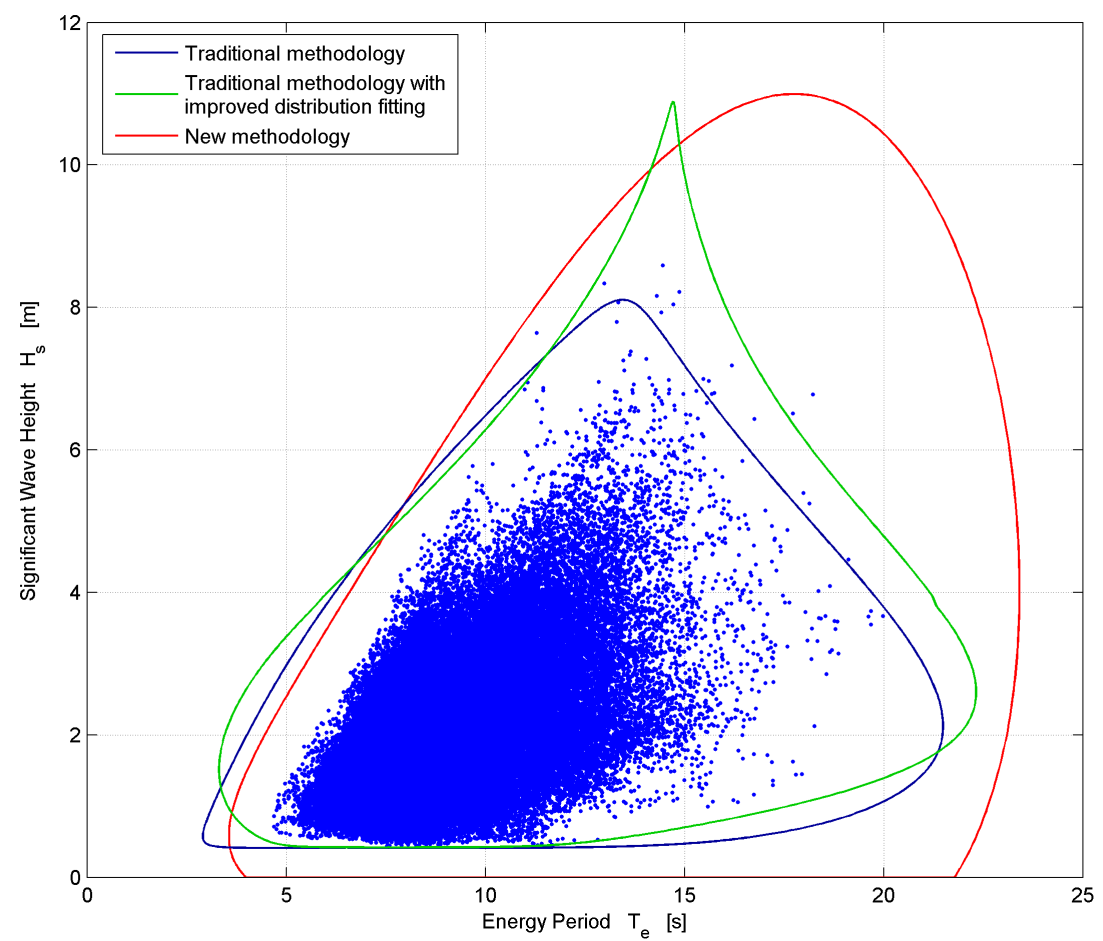

Figure 12: 100-year extreme sea state contour obtained using the traditional methodology (blue), using an updated version of the traditional methodology with better distribution fits (green) and using the new methodologies proposed in this work (red) for NDBC 46212. 